\title{
Effects of vibration rolling on ankle range of motion and ankle muscle stiffness in stroke patients: a crossover study
}

\section{Se-Ju Park}

Nambu University

So-In Lee

Nambu University

Ho-Jin Jeong

Nambu University

Byeong-Geun Kim ( $\nabla$ qudrms_92@naver.com )

Nambu University

\section{Research Article}

Keywords: stroke, vibration rolling, ankle, ROM, stiffness

Posted Date: December 21st, 2020

DOI: https://doi.org/10.21203/rs.3.rs-130805/v1

License: (c) (i) This work is licensed under a Creative Commons Attribution 4.0 International License.

Read Full License 


\section{Abstract}

Background: Vibration stimulation has emerged as a treatment tool to aid spasticity during physical therapy. However, the benefits of vibration rolling (VR) on interventions for stroke patients are unclear. This study aimed to investigate the effect of VR intervention on the range of motion (ROM) and ankle stiffness in stroke patients.

Methods: In this crossover design study, seven stroke patients completed two test sessions (one VR and one non-VR [NVR]) in a randomized order, with $48 \mathrm{~h}$ of rest between each session. Participants completed intervention and its measurements on the same day. The measurements included ankle dorsiflexion and plantarflexion ROM and stiffness of ankle muscles, including the tibialis anterior and gastrocnemius lateral and medial muscles.

Results: After VR, ankle dorsiflexion ROM, gastrocnemius lateral stiffness, and gastrocnemius medial stiffness improved significantly (all $P<0.05$ ). After NVR, only gastrocnemius lateral stiffness improved significantly $(P<0.05)$. Furthermore, compared with the change values for ankle dorsiflexion ROM and gastrocnemius lateral stiffness, VR showed a more significant difference than NVR $(P<0.05)$.

Conclusions: VR improved ankle ROM and muscle stiffness. Therefore, we suggest that practitioners should consider VR as an intervention to increase dorsiflexion ROM and gastrocnemius stiffness in stroke patients.

\section{Background}

Stroke is caused by cerebral infarction and cerebral hemorrhage [1]. Stroke may result in several problems including poor balance and gait, spasticity, weakness, and contractures, as well as sensory and cognitive impairments, all of which require much effort in terms of patient care [2,3]. Among these problems, spasticity results in worse motor function, greater pain and stiffness, and reduced range of motion (ROM) of joints [4,5]. Spasticity can be defined as a sensorimotor disorder related to some level of involuntary muscle activation, and it is a consequence of upper motor neuron syndrome [6,7]. Physical therapy, such as static stretching, transcutaneous electric nerve stimulation, extracorporeal shock wave therapy, electromyography biofeedback, and vibratory stimulation, can be used to treat post-stroke spasticity [812]. However, among the various treatment methods, most vibration stimulation studies have been wholebody vibration studies, and local vibration studies are lacking.

Among the various local vibration tools, vibration rolling (VR) is a combination of foam roller and vibration function. VR has been reported to improve ROM, flexibility, pain, strength, proprioception, balance, and muscle tone in adults [13-15]. Recently, VR has been reported to improve athletic performance in athletes [16]. These effects are necessary not only for adults or athletes but also for stroke patients. Despite this, VR intervention has not yet been studied in stroke patients. 
Therefore, the purpose of this study was to investigate the effect of VR intervention on the ROM and stiffness of the ankle joint in stroke patients and to present the VR as one of the treatment and exercise methods of possible intervention for stroke patients.

\section{Methods}

\section{Participants}

The study was approved by the Institutional Review Board of Nambu University (IRB: 1041478-2020-HR031). Eight stroke patients participated; however, one patient in poor condition was removed during the study. Therefore, seven patients (sex male/female: 6/1; paralyzed side left/right: 3/4; age: $71.43 \pm 9.64$ years, body mass: $67.00 \pm 9.47 \mathrm{~kg}$, height: $170.71 \pm 6.52 \mathrm{~cm}$; onset period: $12.57 \pm 3.10$ month; mini mental state examination, MMSE: $24.86 \pm 0.90$ score; modified Ashworth scale, MAS: $1.21 \pm 0.27$ grade) completed the study. The inclusion criteria were a diagnosis of stroke over 6 months ago, ability to move ankles without assistance, and ankle MAS below Grade 2. The exclusion criteria were as follows: cardiovascular or respiratory disease, orthopedic diseases in the legs, vision or hearing disabilities, and skin diseases of the feet. All participants were informed of the benefits and risks of this study, and written informed consent was obtained from all participants.

\section{Study procedures}

This study was a crossover study. Assessments performed by each participant were assessed in a physical therapy room at the Department of Rehabilitation Medicine, Suwan Medical Center. Prior to the assessment, participants received instruction on how to perform VR and non-VR (NVR) exercises. During this orientation, participants were familiarized with the procedures, assessment tools, and VR equipment of the study. One day after the orientation session, each participant completed two assessment sessions in a randomized order, with $48 \mathrm{~h}$ of rest between each session. Before the assessment session, each participant completed general physical therapy and rehabilitation exercises. Participants rested before the assessment session. An assessment session was conducted in the afternoon. An assessor prepared randomly shuffled sticks (A stick: VR; and B stick: NVR) and sealed each stick in an opaque envelope. Each participant looked for an envelope and opened the envelope to identify the exercise of assignment. The identified exercise was performed first, and the other exercise was performed two days later. The paretic leg of the participants was assessed using ROM of ankle dorsiflexion and plantarflexion, muscle stiffness of the tibial anterior muscle, and medial and lateral gastrocnemius muscles. After the completion of the pre-test assessments, each participant performed the intervention. Each intervention was performed in three sessions for 1 minute per session, and a 30-second rest was taken between each session. Immediately after the intervention, post-test assessments were conducted in the same order as pre-test measures. Participants completed the intervention and its measurements on the same day. One participant in the poor condition was lost to follow-up for the second session. A flowchart of the experimental design is shown in Figure 1.

\section{ROM of ankle}


The ROM of the ankle was measured using a plastic goniometer. The angle of ankle dorsiflexion in the prone position with $90^{\circ}$ of knee flexion was measured. The angle of ankle plantarflexion in the supine position and the ankles outside the bed were measured. The axis of the plastic goniometer was placed on the lateral malleolus. The fixed arm was placed parallel to the line connecting the fibular head, and the moving arm was placed parallel to the line connecting the metatarsal bone of the fifth toe [17]. The measurements were performed by a physical therapist who was blinded to the measured values. The measurer told the recorder when the measurement was complete. The recorder visually confirmed and recorded the measured value. The data used the average of the two measured values. The plastic goniometer showed high interrater reliability $(I C C=0.87)$ and intrarater reliability $($ ICC $=0.91)$ [17].

\section{Muscle stiffness of ankle}

The stiffness of the ankle muscles of the tibialis anterior, gastrocnemius lateral, and medial muscles was measured using the myotonPro (Myoton AS, Tallinn, Estonia). The stiffness of the tibialis anterior in the supine position was measured [18]. The stiffness of the gastrocnemius lateral and medial muscle was measured with the patient in the prone position and with feet hanging over the end of the bed [19]. The measurements were performed by a physical therapist who was blinded to the measured values. The measurer told the recorder when the measurement was complete. The recorder visually confirmed and recorded the measured value. The data used the average of the two measured values. The myotonPro showed high interrater reliability $(I C C=0.93)$ and intrarater reliability $(I C C=0.95)[20,21]$.

\section{Exercise protocols}

\section{VR}

Participants performed VR using a vibrating foam roller (Vyper, Hyperice, Irvine, CA, US). Participants positioned the vibrating foam roller below the gastrocnemius of their paretic side leg. The frequency of VR was $28 \mathrm{~Hz}$, which has been used in many prior studies [13, 22]. Thereafter, patients performed $60 \mathrm{~s}$ of dorsiflexion and plantarflexion of their ankle (Fig. 2). The physical therapist observed and encouraged the patient to continuously move through the entire ROM. Patients engaged in $30 \mathrm{~s}$ of rest in between exercises. Each exercise was performed three times.

\section{NVR}

The exercise protocols were the same as those used for the VR exercise, except vibration (vibration button off).

\section{Statistical analyses}

All data analyses were performed using SPSS version 25 (Chicago, IL, USA). Data are presented as the mean \pm standard deviation (SD). Data were not observed statistically for normality (Shapiro-Wilk's test, $p$ $<0.05$ ), and a few variables were normally distributed. Therefore, nonparametric tests were used. Descriptive statistics were performed for the characteristics of the participants. A Mann-Whitney U test 
was performed to analyze the differences between VR and NVR by comparing the differences between preand post-treatment measurements. The Wilcoxon test was performed to compare pre-and postintervention results in each group. The effect size (Cohen's $d$ ), which is the difference between the pre- and post-means divided by their common SD, was calculated and interpreted as small $(d=0.2)$, medium $(d=$ $0.5)$, or large $(d=0.8)$ to present the magnitude of the effect [23]. The significance level (a) was considered to be $p<0.05$.

\section{Results}

\section{ROM of ankle}

For ankle dorsiflexion ROM, VR showed significant improvement in post hoc test measures $(P<0.05)$ (Table 1) compared with pre-test measures. NVR showed no significant improvement in post hoc test measures $(P>0.05)$ (Table 1$)$ compared with pre-test measures. In addition, compared with the change values, VR showed a more significant difference than NVR $(P<0.05)$ (Fig. 3; A).

For ankle plantar flexion ROM, all groups showed no significant improvement in post hoc test measures ( $P$ $>0.05$ ) (Table 1) compared with pre-test measures. In addition, compared groups in change values, and the two groups showed no significant difference $(P>0.05)$ (Fig. 3; B).

\section{Stiffness of ankle muscle}

For tibialis anterior stiffness, all groups showed no significant improvement in post hoc test measures $(P>$ 0.05 ) (Table 1) compared with pre-test measures. In addition, compared groups in change values, and the two groups showed no significant difference $(P>0.05)$ (Fig. 3; C).

For gastrocnemius lateral stiffness, all groups showed significant improvement in post hoc test measures $(P<0.05)$ (Table 1) compared with pre-test measures. In addition, compared with the change values, VR showed a more significant difference than NVR $(P<0.05)$ (Fig. 3; D).

For gastrocnemius medial stiffness, VR showed significant improvement in post hoc test measures $(P<$ 0.05) (Table 1) compared with pre-test measures. NVR showed no significant improvement in post hoc test measures $(P>0.05)$ (Table 1$)$ compared with pre-test measures. In addition, compared groups in change values, and the two groups showed no significant difference $(P>0.05)$ (Fig. 3; E).

Table 1. Pre-test and post-test measures results 


\begin{tabular}{|c|c|c|c|c|c|c|}
\hline & ariable & Intervention & Pre & Post & Effect & $\mathrm{Z}$ \\
\hline Range & Dorsiflexion & VR & $8.86 \pm 7.128$ & $11.29 \pm 7.296$ & 0.92 & -2.428 \\
\hline Motion & & NVR & $8.43 \pm 6.106$ & $9.29 \pm 6.824$ & 0.49 & $\begin{array}{r}-1.298 \\
(.194)\end{array}$ \\
\hline & $\begin{array}{c}\text { Plantarflexion } \\
\text { (degrees) }\end{array}$ & VR & $68.57 \pm 7.277$ & $68.29 \pm 7.566$ & 0.13 & $\begin{array}{l}-0.343 \\
(.732)\end{array}$ \\
\hline & & NVR & $67.71 \pm 5.823$ & $66.57 \pm 5.159$ & 0.52 & $\begin{array}{l}-1.382 \\
(.167)\end{array}$ \\
\hline Stiffness & $\begin{array}{l}\text { Tibialis } \\
\text { anterior }\end{array}$ & $\mathrm{VR}$ & $480.79 \pm 81.238$ & $488.00 \pm 84.421$ & 0.51 & -1.352 \\
\hline & $(\mathrm{N} / \mathrm{m})$ & NVR & $424.64 \pm 94.385$ & $437.07 \pm 102.782$ & 0.57 & $\begin{array}{l}-1.521 \\
(.128)\end{array}$ \\
\hline & $\begin{array}{l}\text { Gastrocnemius } \\
\text { lateral }(\mathrm{N} / \mathrm{m})\end{array}$ & VR & $349.36 \pm 82.633$ & $326.64 \pm 87.015$ & 0.90 & $\begin{array}{l}-2.371 \\
(.018) *\end{array}$ \\
\hline & & NVR & $337.86 \pm 102.785$ & $359.57 \pm 94.156$ & 0.83 & $\begin{array}{l}-2.197 \\
(.028) *\end{array}$ \\
\hline & $\begin{array}{l}\text { Gastrocnemius } \\
\text { medial }(\mathrm{N} / \mathrm{m})\end{array}$ & $\overline{\mathrm{VR}}$ & $349.79 \pm 47.708$ & $333.29 \pm 42.168$ & 0.83 & $\begin{array}{l}-2.197 \\
(.028) *\end{array}$ \\
\hline & & NVR & $343.93 \pm 79.200$ & $348.57 \pm 92.175$ & 0.19 & $\begin{array}{l}-0.507 \\
(.612)\end{array}$ \\
\hline
\end{tabular}

${ }^{*} P<0.05$, VR: vibration rolling, NVR: non-vibration rolling

\section{Discussion}

This is the first study to investigate the immediate effects of VR combined with dynamic muscle contraction as an intervention to improve ankle ROM and ankle stiffness in stroke patients. In terms of the effects on ankle ROM, VR significantly improved ankle dorsiflexion ROM. In addition, the amount of angle change for dorsiflexion ROM was greatly improved after VR. These results are supported by those of previous studies in which VR increased ankle ROM [22,24]. This effective result is thought to be the result of vibration stimulation leading to an increase in blood flow and temperature, which could provoke ROM improvements [25].

Next, our study revealed that VR significantly decreases gastrocnemius lateral and medial stiffness. These results were similar to those of previous studies in which VR decreased ankle stiffness in athletes [16]. However, as stroke patients experience upper motor neuron syndrome, spasticity occurs as a clinical characteristic of movement disorder. Spasticity can increase muscle stiffness due to exacerbation of stretch reflexes [26]. The stretch reflexes increase in proportion to the speed of movement [27]. This study is thought to suppress the stretch reflex, and the stiffness did not increase because the dynamic movement was slowly maintained during exercise. Additionally, it is thought that the stiffness was improved because the vibration caused a change in the viscoelastic properties of the muscle and increased stretch tolerance $[28,29]$.

Finally, this study showed that improvements in stiffness of the gastrocnemius lateral muscle were significantly better than that of the medial muscle. This is thought to be because the gastrocnemius 
lateral muscle receives direct vibrational stimulation because the hip joint external rotation occurs when the stroke patient is in a supine position.

This study had several limitations. First, the number of participants in the study was small. Second, only one vibration frequency was used. The effects of other vibration frequencies could not be confirmed.

Third, the patient's ankle movement speed and frequency within the intervention time were different. In the future, follow-up studies should be conducted to compensate for these limitations.

\section{Conclusions}

The findings suggest that VR intervention for stroke patients can significantly improve dorsiflexion ROM and gastrocnemius stiffness. Therefore, we suggest that practitioners consider VR as an intervention to increase dorsiflexion ROM and gastrocnemius stiffness in stroke patients.

\section{Abbreviations}

VR: vibration rolling; NVR: non-vibration rolling; ROM: range of motion; MMSE: mini mental state examination; MAS: modified Ashworth scale

\section{Declarations}

\section{Ethics Approval and Consent to Participate}

This study was approved by the Ethics Committee of Nambu University (IRB1041478-2020-HR-031), and it conformed to the ethics guidelines of the Declaration of Helsinki. All the written consents were signed voluntarily. For this study, a privacy regulation applies. This privacy regulation is compliant with the rules of South Korea.

\section{Consent for Publication}

Not applicable.

\section{Availability of Data and Materials}

All data generated or analysed during this study are included in this published article.

\section{Competing Interests}

The authors declare that they have no competing interests.

\section{Funding}

This research received no external funding.

\section{Authors' Contributions}


SJP and BGK were involved in the inception of the idea and study design. SIL and HJJ were responsible for data collection. BGK supervised, SIL and HJJ performed the data analysis. SJP and BGK drafted and finalised the manuscript. All authors have read and agreed to the published version of the manuscript.

\section{Acknowledgements}

The authors want to thank all the patients who agreed to participate and dedicated their time to this study.

\section{Author's Information}

Department of Physical Therapy, Nambu University, Gwangju, South Korea. All authors are physical therapists.

\section{References}

1. Lachance CC, Ford C. Portable stroke detection devices for patients with stroke symptoms: A review of diagnostic accuracy and cost-effectiveness. [Internet]. Ottawa (ON): Canadian Agency for Drugs and Technologies in Health; 2019 Aug. http://www.ncbi.nlm.nih.gov/books/NBK549206/PubMed. Accessed 1 Dec 2020.

2. Mansfield A, Inness EL, Mcilroy WE. Stroke. Handb Clin Neurol. 2018;159:205-28.

3. Li S. Spasticity, motor recovery, and neural plasticity after stroke. Front Neurol. 2017;3:

4. Opheim A, Danielsson A, Alt MM, Persson HC, Sunnerhagen KS. Upper-limb spasticity during the first year after stroke: stroke arm longitudinal study at the University of Gothenburg. Am J Phys Med Rehabil. 2014;93:884-96.

5. Brainin M, Norrving B, Sunnerhagen KS, Goldstein LB, Cramer SC, Donnan GA, et al. Poststroke chronic disease management: towards improved identification and interventions for poststroke spasticity-related complications. Int J Stroke. 2011;6:42-6.

6. Li S, Francisco GE. New insights into the pathophysiology of post-stroke spasticity. Front Hum Neurosci. 2015;10:192.

7. Pandyan AD, Gregoric M, Barnes MP, Wood D, Van WF, Burridge J, et al. Spasticity: clinical perceptions, neurological realities and meaningful measurement. Disabil Rehabil. 2005;27:2-6.

8. Salazar AP, Pinto C, Ruschel Mossi JV, Figueiro B, Lukrafka JL, Pagnussat AS. Effectiveness of static stretching positioning on post-stroke upper-limb spasticity and mobility: Systematic review with metaanalysis. Ann Phys Rehabil Med. 2019;62:274-82.

9. Kwong PW, Ng GY, Chung RC, Ng SS. Transcutaneous electrical nerve stimulation improves walking capacity and reduces spasticity in stroke survivors: a systematic review and meta-analysis. Clin Rehabil. 2018;32:1203-19.

10. Athanasiadis D, Stefas E, Kapsokoulou A, Papathanasiou J, Dionyssiotis Y. Combination therapy for treatment of spasticity in stroke patients: A case study. Curr Health Sci J. 2020;46:180-9. 
11. Vieira D, Silva MB, Melo MC, Soares AB. Effect of myofeedback on the threshold of the stretch reflex response of post-stroke spastic patients. Disabil Rehabil. 2017;39:458-67.

12. Cabanas-Valdés R, Serra-Llobet $P$, Rodriguez-Rubio PR, López-de-Celis $C$, Llauró-Fores $M$, Calvo-Sanz $J$. The effectiveness of extracorporeal shock wave therapy for improving upper limb spasticity and functionality in stroke patients: a systematic review and meta-analysis. Clin Rehabil. 2020;34:114156.

13. Lee CL, Chu IH, Lyu BJ, Chang WD, Chang NJ. Comparison of vibration rolling, nonvibration rolling, and static stretching as a warm-up exercise on flexibility, joint proprioception, muscle strength, and balance in young adults. J Sports Sci. 2018;36:2575-82.

14. Cheatham SW, Stull KR, Kolber MJ. Comparison of a vibration roller and a nonvibration roller intervention on knee range of motion and pressure pain threshold: A randomized controlled trial. $\mathrm{J}$ Sport Rehabil. 2018;1:1-7.

15. Lim JH, Park CB. The immediate effects of foam roller with vibration on hamstring flexibility and jump performance in healthy adults. J Exerc Rehabil. 2019;15:50-4.

16. Lin WC, Lee CL, Chang NJ. Acute effects of dynamic stretching followed by vibration foam rolling on sports performance of badminton athletes. J Sports Sci Med. 2020:19;420-8.

17. Alawna MA, Unver BH, Yuksel EO. The reliability of a smartphone goniometer application compared with a traditional goniometer for measuring ankle joint range of motion. J Am Podiatr Med Assoc. 2019;109:22-9.

18. Saldıran TÇ, Atıcı E, Rezaei DA, Öztürk Ö, Uslu B, Özcan BA, et al. The acute effects of different intensity whole-body vibration exposure on muscle tone and strength of the lower legs, and hamstring flexibility: A pilot study. J Sport Rehabil. 2020;29:1-7.

19. Albin SR, Koppenhaver SL, MacDonald CW, Capoccia S, Ngo D, Phippen S, et al. The effect of dry needling on gastrocnemius muscle stiffness and strength in participants with latent trigger points. $J$ Electromyogr Kinesiol. 2020;55:102479.

20. Feng YN, Li YP, Liu CL, Zhang ZJ. Assessing the elastic properties of skeletal muscle and tendon using shearwave ultrasound elastography and MyotonPRO. Sci Rep. 2018;8:17064.

21. Kelly JP, Koppenhaver SL, Michener LA, Proulx L, Bisagni F, Cleland JA. Characterization of tissue stiffness of the infraspinatus, erector spinae, and gastrocnemius muscle using ultrasound shear wave elastography and superficial mechanical deformation. J Electromyogr Kinesiol. 2018;38:73-80.

22. Lyu BJ, Lee CL, Chang WD, Chang NJ. Effects of vibration rolling with and without dynamic muscle contraction on ankle range of motion, Proprioception, muscle strength and agility in young adults: A crossover study. Int J Environ Res Public Health. 2020;17:354.

23. Lakens $D$. Calculating and reporting effect sizes to facilitate cumulative science: a practical primer for t-tests and ANOVAs. Front Psychol. 2013;4:863.

24. García-Gutiérrez MT, Guillén-Rogel P, Cochrane DJ, Marín PJ. Cross transfer acute effects of foam rolling with vibration on ankle dorsiflexion range of motion. J Musculoskelet Neuronal Interact. 2018;18:262-7. 
25. Veqar Z, Imtiyaz S. Vibration therapy in management of delayed onset muscle soreness (DOMS). J Clin Diagn Res. 2014;8:1-4.

26. Lance JW. The control of muscle tone, reflexes, and movement: Robert Wartenberg Lecture. Neurology 1980;30:1303-13.

27. Knutsson E, Mårtensson A, Gransberg L. Influences of muscle stretch reflexes on voluntary, velocitycontrolled movements in spastic paraparesis. Brain 1997;120:1621-33.

28. Young JD, Spence AJ, Behm DG. Roller massage decreases spinal excitability to the soleus. J Appl Physiol. 2018;124:950-9.

29. Jay K, Sundstrup E, Søndergaard SD, Behm D, Brandt M, Særvoll CA, et al. Specific and cross over effects of massage for muscle soreness: randomized controlled trial. Int J Sports Phys Ther. 2014;9:82-91.

\section{Figures}




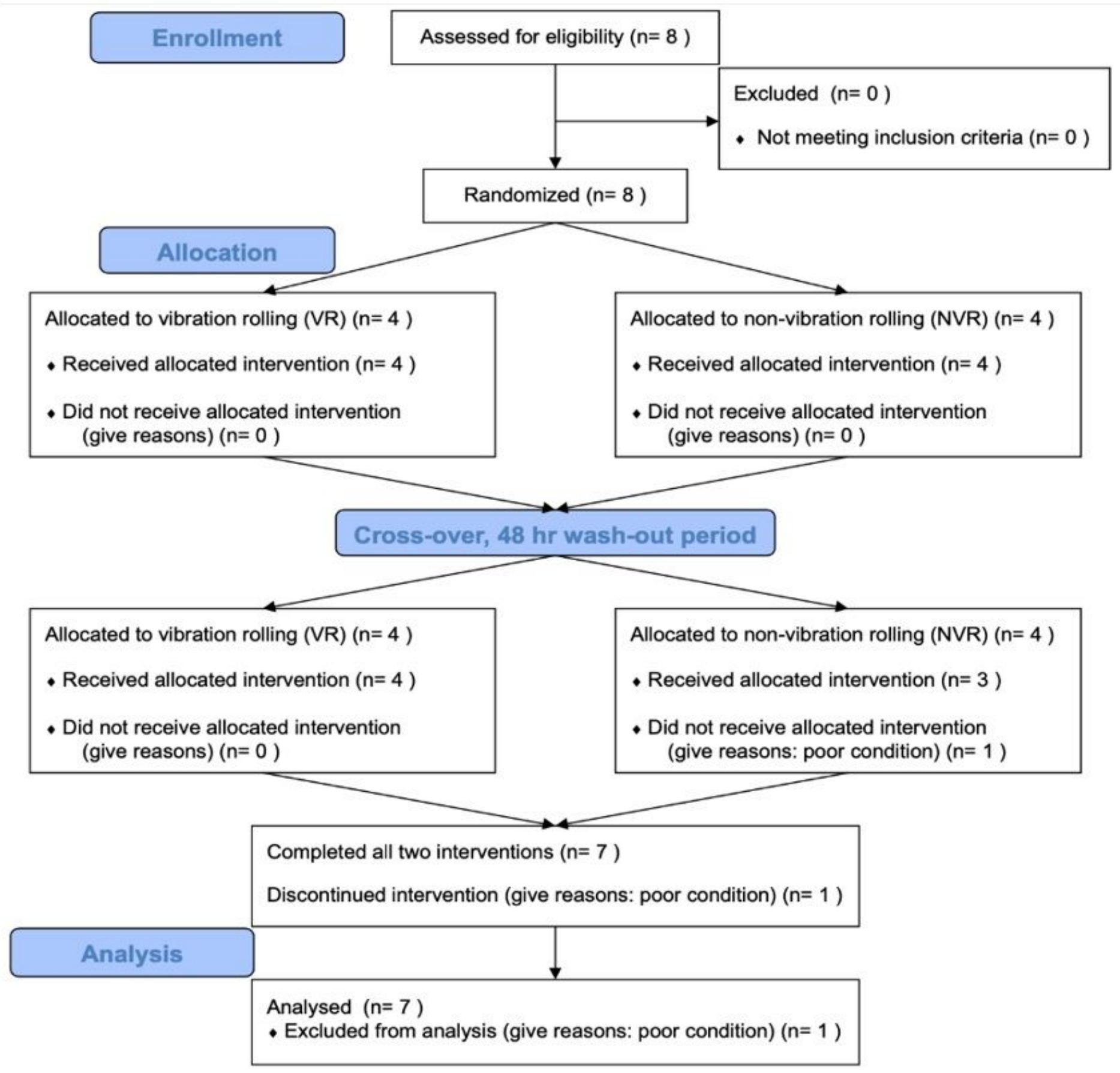

Figure 1

CONSORT flow diagram of the experimental design 


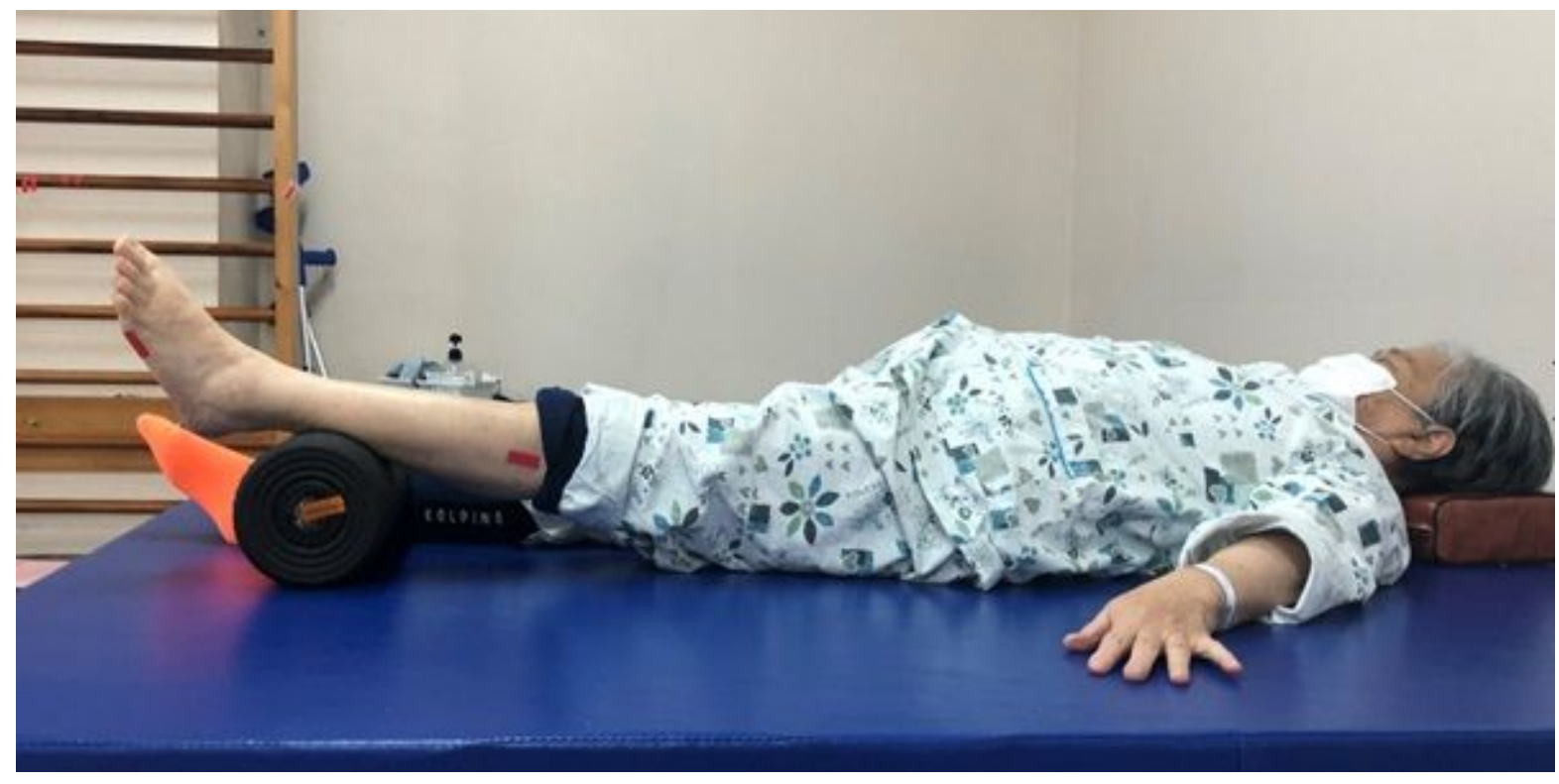

\section{Figure 2}

Vibration rolling on the ankle joint
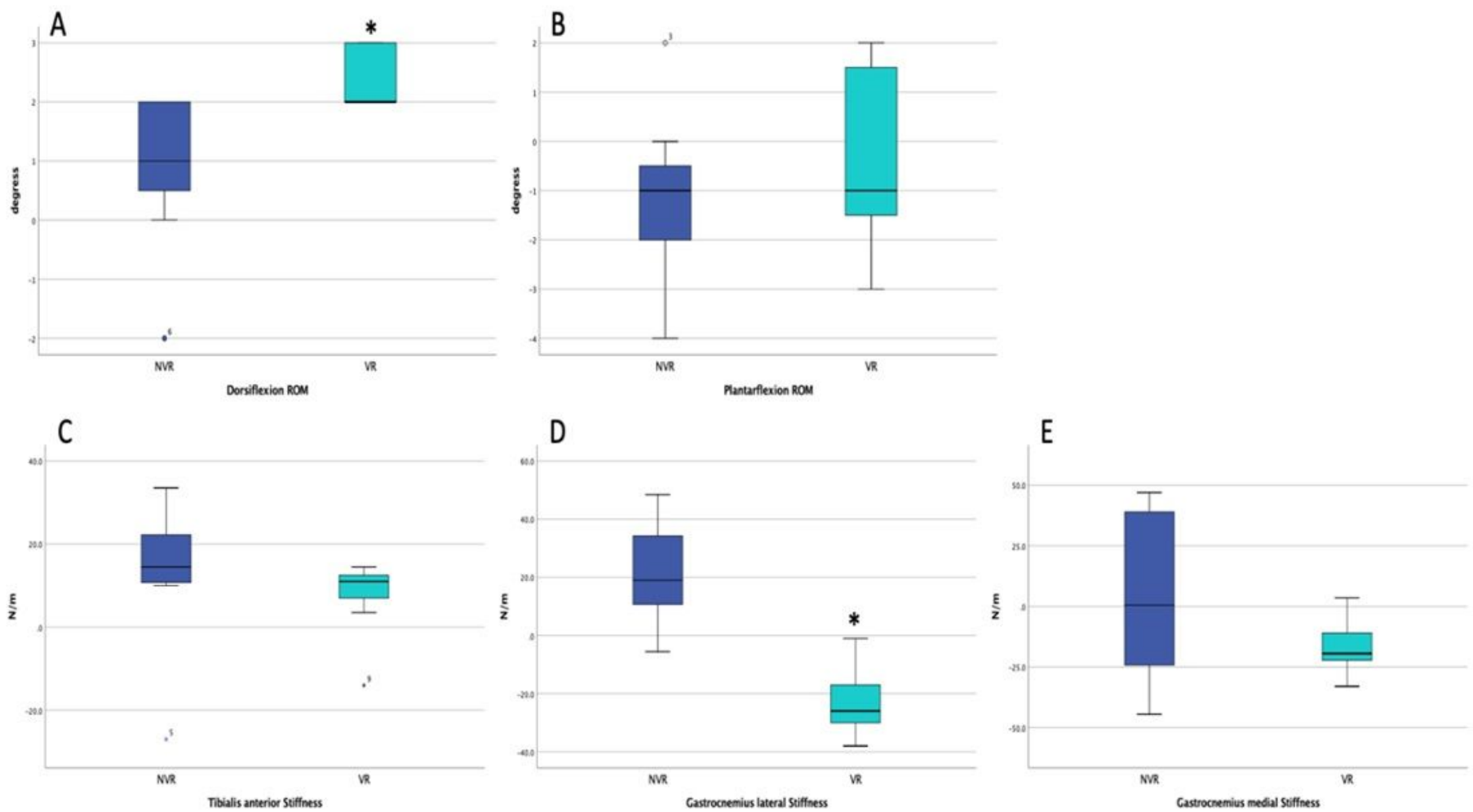

Figure 3

Changes from pre-test to post-intervention in ankle range of motion (ROM) and ankle muscle stiffness after vibration rolling (VR) and non-vibration rolling (NVR) * Change is statistically significant at $\mathrm{p}<0.05$. 